INPLASY

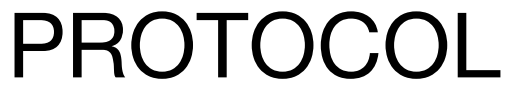

To cite: Zhang et al. Efficacy of combination of variant pills of six ingredients with rehmannia and nitroimidazoles in the treatment of atrophic vaginitis: a systematic review and metaanalysis. Inplasy protocol 2021120018. doi:

10.37766/inplasy2021.12.0018

Received: 03 December 2021

Published: 03 December 2021

Corresponding author:

Yi Zhou

zhoumen1123456@163.com

Author Affiliation:

College of Basic Medicine,

Chengdu University of

Traditional Chinese Medicine.

Support: None.

Review Stage at time of this submission: Preliminary searches.

Conflicts of interest: None declared.

\section{Efficacy of combination of variant pills of six ingredients with rehmannia and nitroimidazoles in the treatment of atrophic vaginitis: a systematic review and meta-analysis}

Zhang, Y1; Mo, J2; Han, L3; Tang, H4 Zheng, C5; Xie, Y6.

Review question / Objective: Is combination of variant pills of six ingredients with rehmannia and nitroimidazoles effective for atrophic vaginitis? Can combination of variant pills of six ingredients with rehmannia and nitroimidazoles be a complementary and alternative therapy for atrophic vaginitis? P: Participant: Elderly women diagnosed with atrophic vaginitis; I: Intervention: combination of variant pills of six ingredients with rehmannia and nitroimidazoles; C:Comparison: nitroimidazoles or with oestrogen; 0:Outcome: effective rate; S: Study design: RCTs.

Condition being studied: Postmenopausal women with vaginitis is known as atrophic vaginitis disease, the main clinical manifestations include: increased vaginal secretions, painful sexual intercourse and vulva itching. The disease is difficult to cure and has high incidence rate. The main treatment is using antibiotic, estriol, lactobacillus. In recent years, there are more and more clinical reports on the treatment of atrophic vaginitis by combination of variant pills of six ingredients with rehmannia and nitroimidazoles, but there is still a lack of effective evidence.

INPLASY registration number: This protocol was registered with the International Platform of Registered Systematic Review and Meta-Analysis Protocols (INPLASY) on 03 December 2021 and was last updated on 03 December 2021 (registration number INPLASY2021120018).

\section{INTRODUCTION}

Review question / Objective: Is combination of variant pills of six ingredients with rehmannia and nitroimidazoles effective for atrophic vaginitis? Can combination of variant pills of six ingredients with rehmannia and nitroimidazoles be a complementary and alternative therapy for atrophic vaginitis? $P$ : Participant: Elderly women diagnosed with atrophic vaginitis; I: Intervention: combination of variant pills of six ingredients with rehmannia and nitroimidazoles; C:Comparison: nitroimidazoles or with oestrogen; 
O:Outcome: effective rate; S: Study design: RCTs.

Rationale: Variant pills of six ingredients with rehmannia is a series of traditional Chinese medicine prescriptions, its main components include: cornus officinalis, ripe rehmannia officinalis, Chinese yam, Alisma orientalis, cortex danpi, tuckahoe, etc. The main effect is nourishing the kidney Yin, removing the fire in the patient's body. The general effect of dogwood is to supplement the liver and kidney, strengthen the kidney Yang, but also to smooth and complement the blood. The general effect of cooked rehmannia is to fill lean pulp. It can nourish blood and Yin, and reduce the side effects of Yin deficiency and Yang hyperactivity type. The general functions of Chinese yam are to strengthen spleen and kidney, enhance resistance, nourish qi and Yin. Some scholars believe that Chinese yam also has the effect of anti-aging. Tangpi can cool blood and clear heat. Poria cocos can inhibit the growth of bacteria. Nitroimidazoles can restrain the breeding of vaginal bacteria. By combining variant pills of six ingredients with rehmannia with nitroimidazoles, the vaginal environment can be improved, bacterial growth can be inhibited and atrophic vaginitis can be cured.

Condition being studied: Postmenopausal women with vaginitis is known as atrophic vaginitis disease, the main clinical manifestations include: increased vaginal secretions, painful sexual intercourse and vulva itching. The disease is difficult to cure and has high incidence rate. The main treatment is using antibiotic, estriol, lactobacillus. In recent years, there are more and more clinical reports on the treatment of atrophic vaginitis by combination of variant pills of six ingredients with rehmannia and nitroimidazoles, but there is still a lack of effective evidence.

\section{METHODS}

Search strategy: Six electronic databases, including China National Knowledge Infrastructure (CNKI), Wanfang Data,
Chinese Scientific Journals Database (VIP), PubMed, EMBASE, and Cochrane Library were searched from inception to December 2021 for identifying eligible studies. No restriction on language or publication status was imposed. Following terms were used in a combination for the electronic search: atrophic vaginitis, senile vaginitis, Postmenopausal vaginitis, pill of six ingredients with rehmannia, *nidazole, randomized control, randomization, randomized clinical trials, RCT, and trials. Any inconsistency was solved by a third reviewer. Manual searches were performed to identify relevant studies in the reference lists of the included studies.

Participant or population: Patients who were diagnosed with atrophic vaginitis (as diagnosed by a clinician, or using any recognized diagnostic criteria). In the same study, patients in different intervention groups had no significant imbalance in baseline conditions such as age, gender and course of disease, and were comparable.

Intervention: The treatment group received combination of variant pills of six ingredients with rehmannia and nitroimidazoles therapies. Herbal fumigation, Baofukang suppository, and other antibiotic will be excluded.

Comparator: Nitroimidazoles, or nitroimidazoles combined with estrone.

Study designs to be included: Randomized controlled trials (RCTs)Randomized controlled trials (RCTS) of combination of variant pills of six ingredients with rehmannia and nitroimidazoles therapies for the treatment of atrophic vaginitis, regardless of the time of publication or field of study, regardless of whether hidden or blind allocation was involved. Nonrandomized controlled trials, such as animal trials, reviews, conferences and papers, clinical case reports, and personal experience, are excluded.

Eligibility criteria: The inclusion criteria were prespecified as types of participants: patients diagnosed with atrophic vaginitis 
using any recognized diagnostic criteria, regardless of age, gender, source of cases, duration of disease, ethnicity, or nationality; (2) types of interventions: combination of variant pills of six ingredients with rehmannia and nitroimidazoles clearly stated in the trial group either alone or in combination with other treatments; no restriction was imposed on the administration mode, dosage, and course of treatment; (3) types of comparator(s)/ control: patients treated with nitroimidazoles or combine with estrogen; (4) types of outcome measures: The main effective rate, recurrence rate, symptom score of vagina, Vaginal ph, cleanliness of vagina,ect.(5) types of study: RCT. Exclusion criteria included (1) non-RCTs, reviews, animal-based research, conference proceedings, and literature review; (2) unclear diagnostic criteria and outcome measurements; (3) unable to get original data; (4)duplicated publications; and (5) other TCM treatments involving acupuncture and massage.

Information sources: The Cochrane Central Register of Controlled Trials (CENTRAL), PubMed, EMBASE, CBM,CNKI,VIP, WanFang will be searched for relevant information, updated to December 2021 without any restriction of language or publication date. To achieve comprehensive information, we will contact with authors, check the trial registers, or search grey literature.

Main outcome(s): The main effective rate, recurrence rate, symptom score of vagina, ph of vagina, cleanliness of vagina, ect. The minor outcome is effective rate of main symptoms : 1.Vaginal itching efficiency 2. Dry and pain vagina efficiency 3.abnormal leukorrhea efficiency.

Additional outcome(s): Additional outcomes are the effective rate of main symptoms of Climacteric syndrome.

Quality assessment / Risk of bias analysis: Two reviewers will evaluate the bias risk of included studies according to Cochrane Handbook 5.1.0. Whether the allocation scheme is hidden; Whether subjects and researchers are blind; Whether the evaluation of the outcome of blind; Whether the result data is complete; Whether research findings are selectively reported; Other sources of bias .According to the evaluation results, use Review Manager 5.2 software to make the bias risk map of the included study.

Strategy of data synthesis: For each trial, we will present outcome data as point estimates with mean and standard deviation for continuous outcomes and risk ratios (RRs) with corresponding 95\% confidence intervals (Cis for dichotomous outcomes. RevMan will be used for direct meta-analysis.Using the Review Manager Software (Revman 5.2). We used fixedeffect meta-analysis if trails were sufficiently similar with no significant heterogeneity. If we had found heterogeneity, we planned to explore the reason by sensitivity analysis followed by random-effect if required. For dichotomous data, we presented the primary outcome of remission of atrophic vaginitis using risk ratios (RR) with $95 \%$ confidence intervals (CI). For continuous data, we would calculate mean difference if the results were measured in the same way between trails. If studies used different methods to assess the same outcome, we would have calculated standardized mean difference to combine trails. As to assessment of reporting biases, we would have generated a funnel plot and visually inspected for asymmetry when the studies are more than ten. Publication bias also was determined using Egger's test to funnel plot asymmetry.

Subgroup analysis: We used the I-squared statistic to apply tests of heterogeneity between trails. When the levels of heterogeneity among the trails exceeded $50 \%$, we would have explored the reason by subgroup analysis and sensitivity analysis followed by random-effect if required. For trails using different treatment methods, we planned to conduct subgroup analysis to assess the effects of different interventions. 
Sensitivity analysis: Sensitivity analysis will be performed for the total effective rate, recurrence rate ,ph of vagina, cleanliness of vagina, and the effective rate of main symptoms : 1.Vaginal itching efficiency 2. Dry and pain vagina efficiency 3.abnormal leukorrhea efficiency.

Language: No restriction on language was imposed.

Country(ies) involved: China.

Keywords: variant pills of six ingredients with rehmannia; nitroimidazoles; atrophic vaginitis; systematic review; meta-analysis.

Contributions of each author:

Author 1 - Yiyi Zhang.

Author 2 - Jingwen Mo.

Author 3 - Lingling Han.

Author 4 - Huiling Tang.

Author 5 - Chengwen Zheng.

Author 6 - Yujia Xie. 